\title{
High-temperature injection spectroscopy of deep traps in CdTe polycrystalline films
}

\author{
A.S. Opanasyuk, N.N. Opanasyuk, N.V. Tirkusova \\ Sumy State University, 2, Rymskyi-Korsakov str., 40007 Sumy, Ukraine \\ Phone: +380 (542) 334058, 335323, fax: +380 (542) 334058, \\ E-mail: denisov@ssu.sumy.ua,denisov@ssu.sumy.ua,nadtir@ssu.sumy.ua
}

\begin{abstract}
The paper provides the direct experimental method to determine the localized state energy distribution function for semiconducting solid materials based on space-charge-limited current-voltage characteristics. The current-voltage characteristics would be obtained under the random temperatures. The Tikhonov regularization method was used to solve the Fredholm 1st rank equation. The method developed in this research was used for the study of deep traps in CdTe polycrystalline films obtained in quasi-closed-tube on the conducting substrate. In the bend gap of the material, some traps were traced, which can be described by the close to Gaussian distribution parameters as well as by the parameter of energy disorder $=0.015-0.04 \mathrm{eV}$. The research shows that the trap concentration and depend on the physical and technological conditions of the obtained films, while the energy of the traps depends on the impurity-defective material structure.
\end{abstract}

Keywords: CdTe polycrystalline films, space-charge-limited currents, deep traps.

Paper received 27.12.02; revised manuscript received 10.09.03; accepted for publication 11.12.03.

Cadmium telluride is one of the most promising materials of $\mathrm{A}^{\mathrm{II}} \mathrm{B}^{\mathrm{IV}}$ group because it can be used in the production of many high-quality devices for solar power engineering, optical electronic, nuclear energetic industry, etc. The necessity to control and manage the electrical and optical characteristics of CdTe crystals and films caused the growth of the researchers' interest to the defective structure of the materials. The scientists want to know how the defectiveness of structure affects the physical characteristics of the materials. Although many works and investigations have been done in the field, the energy spectrum of the localized states (LS) in the band gap (BG) of CdTe has not been studied sufficiently. Film samples produced by different ways of vacuum condensation are described the least.

The method for the analysis of the space-charge-limited currents (SCLC) stationary current-voltage characteristics $(\mathrm{CVC})$ is one of the most common methods for the investigation of deep traps (DT) in semiconductive materials of the CdTe type. With the simplicity of the necessary equipment, the method provides strong sensitivity to low concentrations of LS [1-3].
But used in its traditional form, the SCLC CVC method gives some errors that effect in less amount of information as well as distortion of some experimental results [4]. Meanwhile, some researches [5-6] provide methods that allow to avoid the errors of the traditional SCLC CVC method and receive more detailed information about DT spectrum in the BG of the materials immediately from the differentiation of experimental curves. This is the method of injection spectroscopy (IS) or the way to solve the problem under the investigation 'from the opposite.'

Despite its vivid advantages over the traditional CVC analysis methods, the IS method is still less known and rarely used due to its complicated processing of the experimental curves. Although, some works [7-8] showed its high sensitivity and effectiveness for the study of DG in organic materials. Many researches [7-9] that used this method, as a rule, used low temperatures, while in real applications, most of the data are obtained by the close to room temperatures. As demonstrated in [10-13], such inconsistency results in the discrepancy of the picture of the gaps energetic distribution. To avoid this discrepancy, the [12] recommends high-temperature IS method. The suggested method allows to determine the 


\section{A.S. Opanasyuk et al.: High-temperature injection spectroscopy of deep traps ...}

function of the energy LS distribution immediately from the experimental curves without considerable errors. This work gives the results of the energetic DT distributions in high-resistant polycrystalline CdTe films obtained from the real conditions; the work also provides the equations for the DT distributions in the samples.

Cadmium telluride films on the conductive non-oriented substrate were obtained in a VUP-5M quasi-closedvacuum tube within a wide range of temperatures on the substrate and evaporator $\left(T_{S}=100-550^{\circ} \mathrm{C}, T_{E}=550\right.$ $800^{\circ} \mathrm{C}$ ). Glass-ceramic plates with a molybdenum layer (or some alternative conductive material) were used as substrate. The substrate layer was produced by the electronic-ray evaporation method. The layers of $\mathrm{Au}$ or $\mathrm{Ag}$ were used for the surface contact. These layers were produced by the vacuum evaporation method and permit the substrate injectivity in the $p$-type conductive materials. Standard methods were used to investigate the shadow CVC under different temperatures and sandwich-structure of the $\sigma-T$ dependencies in vacuum. The differential microscopy method developed in [14-15] was used to identify the charge transfer caused by the through-thesample currents. This method permits to separate the adjoining from competitive charge transfers in the structures as well as to determine the high-poled charge transfers. The simultaneous analysis of $j-U, \eta-U$ and $d(\lg \eta) / d(\lg U)-U$ of $(\eta=d(\lg j) / d(\lg U))$ dependences was applied.

It was determined that within the $T_{S}=350-550^{\circ} \mathrm{C}$ range, the films under investigation have one-phase sphalerit column type structure and a grain seed size that is the film thick and equals 0.1 to $(10-20) \mu \mathrm{m}$. The film resistance equals $5 \cdot 10^{6}-10^{11} \mathrm{Ohm} \cdot \mathrm{cm}$. Within the given condensation temperature range, the CVC of the polylayer structures was found through the monopolar injection from the catching terminal. The corresponding SCLC $\mathrm{CVC}$ were investigated by the low- and high-temperature approximation IS method.

The main equations from the IS method in $[5-6,12]$ are

$$
\begin{aligned}
& n_{f L}=\frac{\eta}{2 \eta-1} \frac{j L}{e \mu U}, \\
& n_{s L}=\frac{\rho_{L}}{e}=\frac{2 \eta-1}{\eta} \frac{\eta-1}{\eta}\left[1-\frac{\eta^{\prime}}{\eta(2 \eta-1)(\eta-1)}\right] \frac{\varepsilon \varepsilon_{0} U}{e L^{2}},
\end{aligned}
$$

where $j$ - through the sample current density; $U$ - an applied voltage; $e$ - an electron charge; $\mu$ - mobility of the charge carrier in the material; $L-$ a sample thickness; $\varepsilon_{0}$ - a dielectric constant; $\varepsilon$ - the material dielectric permittivity; $n_{f L}$ - the injection free carriers concentration on the anode (index $L$ will be omitted in the further equations ; $n_{s L}$ - the factual concentration of the carriers trapped near the anode; $\rho_{L}$ - the space charge density in the material (on the anode) that causes the limited through the sample current.
The following equation allows to determine the DP energy distribution based on the material free charge carriers concentration and the Fermi-Level ratio.

$$
E_{F}=k T \ln \frac{N_{c(v)} e \mu}{L}+k T \ln \frac{j}{U}+k T \ln \frac{\eta}{2 \eta-1},
$$

where $E_{F}$ - the location of Fermi's quasi-level in injection, $k$ - is Boltzman's constant, $T$ - the temperature of measurement, $N_{C(V)}$ - effective density of free states in conduction band (valence band) of the material.

Function of storage change distribution, localized on deep centers will get in the way of differentiation correlation (2) in energy

$$
\frac{d n_{s}}{d E_{F}}=\frac{1}{k T} \frac{2 \eta-1}{\eta}\left\{1+\frac{3(\eta-1) \eta \eta^{\prime}-\eta \eta^{\prime \prime}+3 \eta^{\prime 2}}{\eta^{2}\left[(2 \eta-1)(\eta-1)-\eta^{\prime} / 2\right]}\right\} \frac{\varepsilon \varepsilon_{0}}{e L^{2}} U
$$

The assumptive result of the application of a low-temperature method reads that the function of the LS density distribution for the energy $h(E)=\frac{d N_{t}}{d E}$ coincides with the function that describes the distribution of the localized carriers. But it results in the errors for the traps distribution shape as well as for the traps concentration $\left(N_{t}\right)$ and localization depth $\left(E_{t}\right)[6-7,10-13]$. At optional temperatures the $h(E)$ function can be found from the convolution

$$
\frac{d n_{s}}{d E_{f}}=\int_{E} h(E) \frac{d f\left(E-E_{f}\right)}{d\left(E-E_{f}\right)} d E
$$

where $f\left(E-E_{F}\right)$ is the Fermi-Dirak function.

The solution of equations (3),(4) and (5) allows to find the function $h(E)$ in $\mathrm{BG}$ of the material directly from the experimental SCLCC CVC at a tolerant temperature. For the purpose, it is necessary to determine 3 derivatives at each $j-U$ interdependence in double logarithmic coordinates. Mathematically this task comes to a smoothing cubic spline, that approximates the experimental data, its differentiation in nodes, followed with the finding of the $h(E)$ function from convolution (5).

Physically the scanning ability of the energy trap distributions by the charge carriers injection can be conditioned as follows: when the external voltage is increased, the Fermi quasi-level starts moving in the BG zone; at this condition, the LS are filled with free charge carriers, and change of the conductivity structure occurs. Due to the interdependences between the data that can be experimentally measured $(j, U)$ and those that characterize the traps distribution, $\left(E_{F}, \rho\right)$ each CVC point gives information about the corresponding area of the LS energy spectrum. Thus, in contrast to the existing integral methods, the IS method gives more complete information about trap spectrum in the material. 


\section{A.S. Opanasyuk et al.: High-temperature injection spectroscopy of deep traps ...}

Since the task of finding $h(E)$ function from convolution (5) is not correct, the Tihonov regularization method [16] was used for its solution. The choice of the regularization parameter $\alpha$ was made via the discrepancy method and by the finding of the quasi-optimal parameter.

To check the correctness of the results got via the hightemperature IS method used for the reconstruction of the traps distribution from the experimental CVC, we applied the computer modeling process that included first direct and then "from the opposite" methods of the problem solution. The modeling was applied to the CdTe deep state parameters and physical constants. The energy of traps localization was measured from the edge of the conductivity zone or for the valency zone.

The theoretical CVC SCLC were received from the direct problem solution, where the input distributions (deep state distributions in the BG of the material) were a given data, and the equations presented in [10-12] were used. At the next step, the theoretical curves were processed via the IS method (3)-(5) equations. In the result, the energy DT distributions (the output distributions) were obtained again. Because the procedure of the theoretical CVC is correct, the concision of the input and output distributions allows to justify the high-temperature IS method as valid for the reconstruction of the traps distribution shape.

Fig. 1 illustrates the results of the modeling for one or two trap distributions in the $\mathrm{BG}$ of the material that has been described by the double exponential distribution or Gauss Functions. Fig. 1 footnote gives the parameters of the model distributions and the material constants that were used for the computations. It is important to say that the calculations were performed for the energy-narrow trap distributions that are typical for polycrystalline materials.

The results of the modeling showed that the shape of the $h(E)$ function is almost exactly re-produced by the IS high-temperature method even when the LS distributions are too narrow and close to $\sigma=0.015 \mathrm{eV}$; where $\sigma$ is a parameter by the Gauss Function. The traps concentration error does not exceed (3-5)\%, while the level localization energy (maximum of the $h(E)$ function) is calculated precisely. In the case of more diffuse distributions, their shape lines error decreases noticeably. What makes this method peculiar is that when applied, it produces the mono-energy levels as very narrow distributions $(\sigma<$ $<0.015 \mathrm{eV}$ ) that are described by the Gauss Function, because for the integral equations, the unknown function is assumed as continuous. If the low-temperature method is applied, as in [11-12], the mono-levels are re-constructed as diffuse curves with the semi-width $\approx 3.5 \mathrm{kT}$ ( $\sigma=0.091 \mathrm{eV}$ by the room temperature).

A typical experimental CVC curve and the results of the traps distribution shape reconstruction in one of the samples got via the high-temperature IS method are pictured in Fig. 2 a, b. To verify the viability of the results for the curve, the $j-U$ sandwich-structures were checked several times in some cases. CVC peculiar properties are noticeable on the $\eta-U$ dependence that demonstrates a
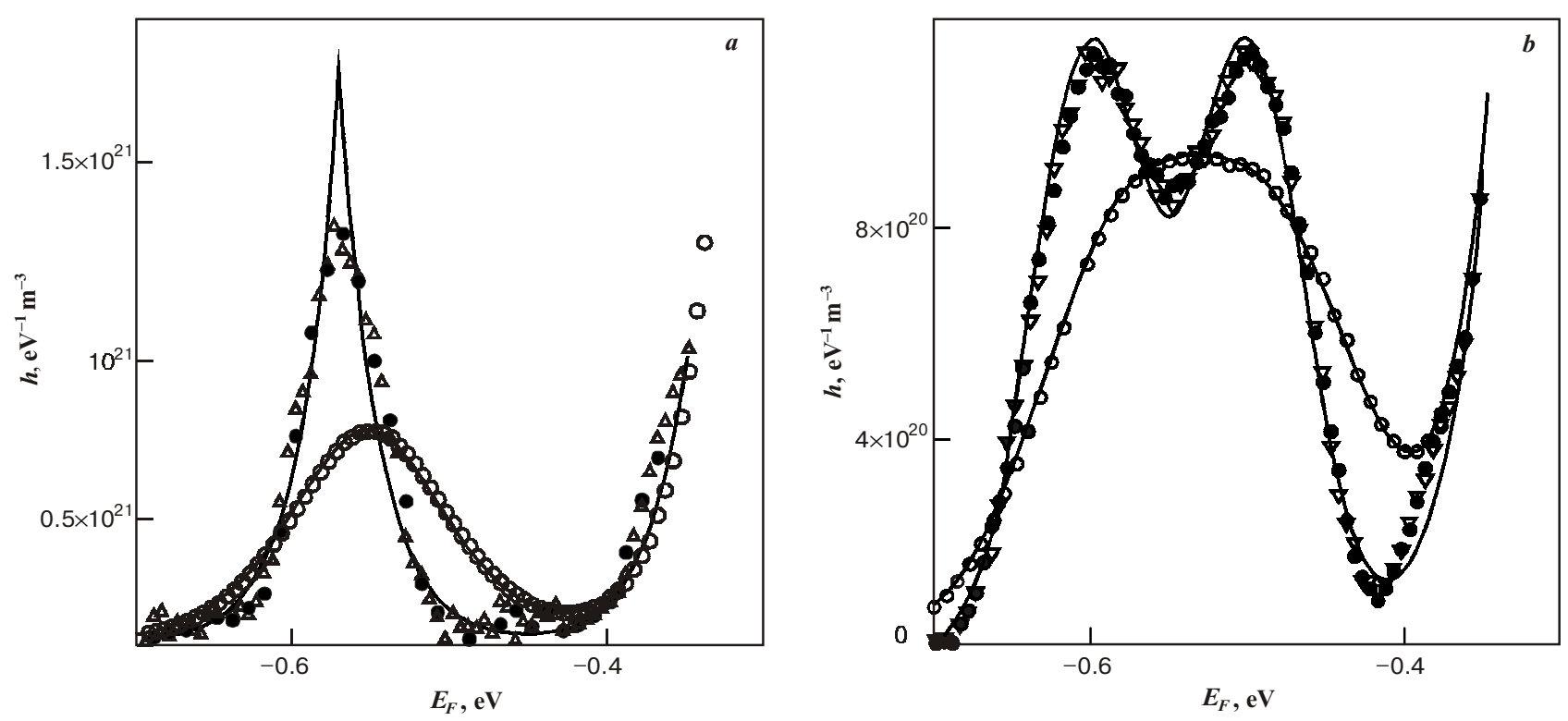

Fig. 1. Localized states distribution in the $\mathrm{BG}$ of the material for:

a) double exponential distribution, where $E_{t}=-0,57 \mathrm{eV}, N_{t}=10^{20} \mathrm{~m}^{-3}, k T_{c}=0,025 \mathrm{eV}, g=2$ (factor of the degeneration); b) two Gauss distributions, where $E_{t 1}=-0,6 \mathrm{eV} ; E_{t 2}=-0,5 \mathrm{eV} ; N_{t}=10^{20} \mathrm{~m}^{-3} ; \mathrm{s}=0,025 \mathrm{eV} ; g=1$.

Input distribution $h(0)$ - a solid line; output distribution: $O$-calculation with the low-temperature method IS; calculation with the high-temperature method IS: - - discrepancy method; $\nabla$ - quasi-optimal parameter method. 


\section{A.S. Opanasyuk et al.: High-temperature injection spectroscopy of deep traps ...}
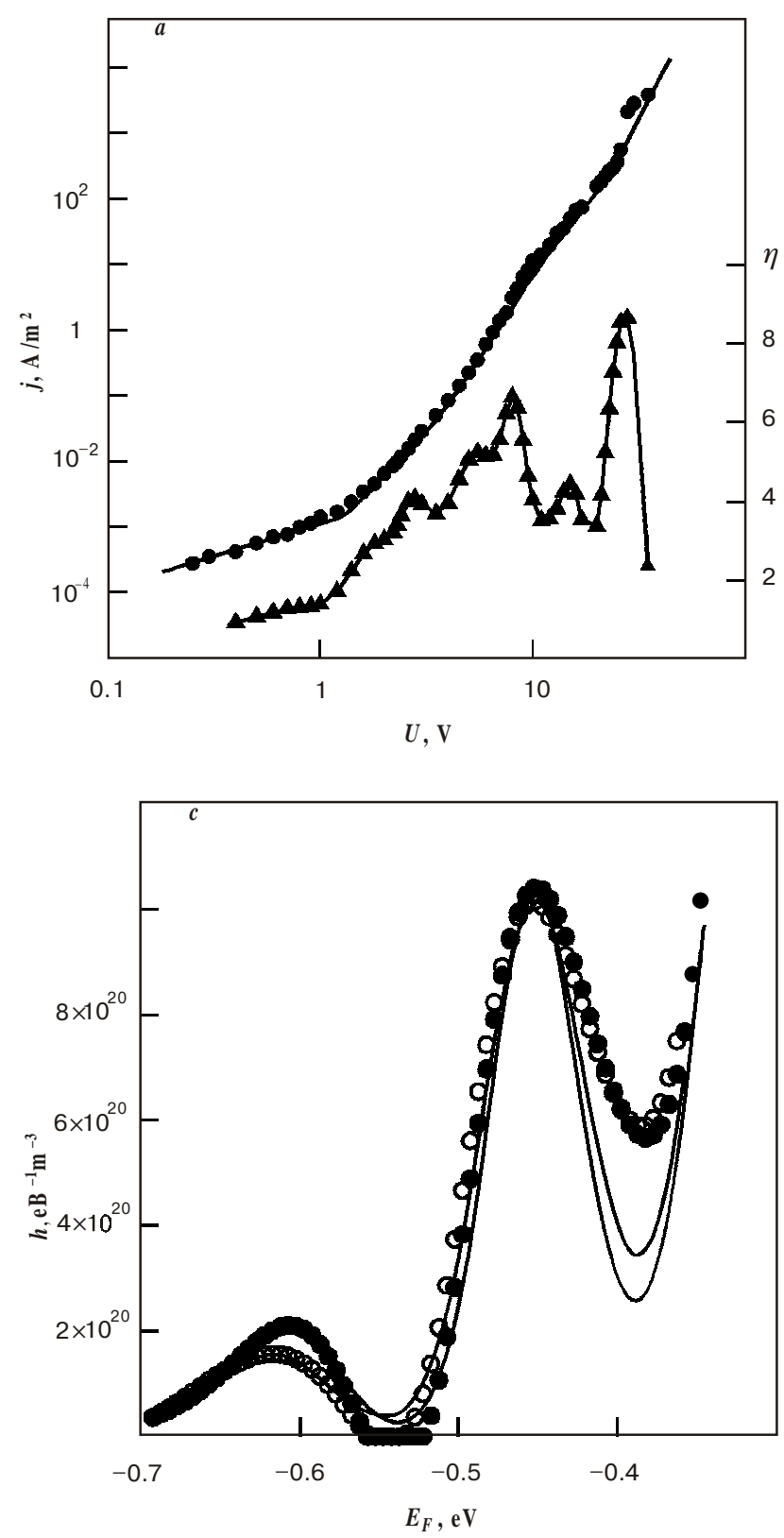

fine structure of these curves. As the figure illustrates, the CVCs from the first and second surveys almost coincide.

Fig. $2 c$ illustrates the results of the IS high-temperature experimental curves processing. It is clearly noticeable that the DT distributions got from the two different CVC and by close temperature parameters coincide well. To verify the traps distribution shape got from the experiment, Fig. $2 c$ includes the distributions described by the Gauss Function. The figure also shows that CdTe polycrystalline films have the $\mathrm{BG}$ trap distributions close to the Gaussian ones with small s. Similar distributions were noticed in polycrystalline $\mathrm{Pb}_{2} \mathrm{Bi}_{6} \mathrm{O}_{11}, \mathrm{Y}_{2} \mathrm{O}_{3}$ and other samples obtained by the thermo-stimulated currents in [18]. Quasi-continuous energy-dependent distribution of traps in the vacuum-condensed films may be influenced by the static dispersion of the charge carriers' polarized

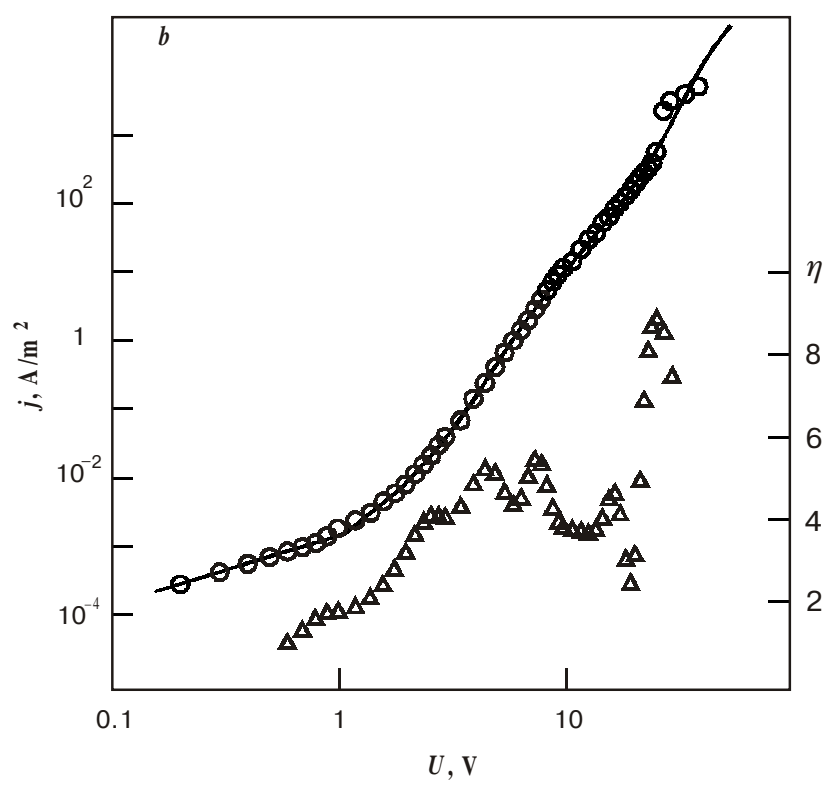

Fig. 2. CVC and its derived function $\eta(U)$ for sandwich-structure Ag-CdTe-SnO ${ }_{2}\left(L=1.9 \cdot 10^{-5} \mathrm{~m}, S=7 \cdot 10^{-6} \mathrm{~m}^{2}\right)$, and the traps distribution in the material $\mathrm{BG}$.

a) $-j(U) ; \boldsymbol{\Delta}-h(U)$ - first measuring; b) $\bigcirc-j(U) ; \Delta-h(U)-$ second measuring; c) energy-dependant traps distribution from the high-temperature IS method ( - first measurement; $\bigcirc$ second measurement); Gaussian distributions are given for comparison - the solid line.

energy caused by the fluctuations of the crystal lattice of material.

The calculations of parameters of the deep centers in tested samples are shown in the table. The depth of the traps was determined at its maximum on the $h(E)$ dependence, and their concentration was determined from the area corresponding to its curve. As it is shown in the table, the errors in the finding of traps parameters by different measurements do not exceed the errors of IS method [11].

The studies have shown that $\mathrm{CdTe}$ films are characterized by DT in BG with $E_{t 1}=0.68-0.70 \mathrm{eV} ; E_{t 2}=0.60$ $0.63 \mathrm{eV} ; E_{t 3}=0.56-0.57 \mathrm{eV} ; E_{t 4}=0.51-0.53 \mathrm{eV}$; $E_{t 5}=0.45-0.46 \mathrm{eV} ; E_{t 6}=0.39-0.41 \mathrm{eV}$ and $N_{t}=10^{18}$ $10^{20} \mathrm{~m}^{-3}$. The wide spectrum of the traps was, probably, conditioned by the fact that LS were investigated in the 
A.S. Opanasyuk et al.: High-temperature injection spectroscopy of deep traps ...

Table. The DT parameters found in the CdTe films by high temperature IS method

\begin{tabular}{ccccccc}
\hline \hline Number of sample & $L, \mu \mathrm{m}$ & $T_{S}{ }^{\circ} \mathrm{C}$ & $T_{E}-T_{S}{ }^{\circ} \mathrm{C}$ & $E_{t}, \mathrm{eV}$ & $N_{t}, \mathrm{~m}^{-3}$ & $\sigma, \mathrm{eV}$ \\
\hline 1 & 8 & 470 & 280 & 0.63 & $4.4 \cdot 10^{19}$ & 0.030 \\
\hline 2 (first measurement) & 19 & 475 & 200 & 0.61 & $1.7 \cdot 10^{19}$ & 0.031 \\
& & & & 0.45 & $7.3 \cdot 10^{19}$ & 0.028 \\
\hline 2 (second measurement) & 19 & 475 & 200 & 0.62 & $1.5 \cdot 10^{19}$ & 0.035 \\
& & & & 0.45 & $8.1 \cdot 10^{19}$ & 0.032 \\
\hline 3 & 12 & 475 & 220 & 0.68 & $7.8 \cdot 10^{18}$ & 0.023 \\
& & & & 0.62 & $1.5 \cdot 10^{19}$ & 0.023 \\
& & & & 0.53 & $6.1 \cdot 10^{19}$ & 0.027 \\
\hline 4 (monocrystal) & 11 & & & 0.62 & $4.6 \cdot 10^{18}$ & 0.019 \\
& & 480 & & 0.52 & $1.3 \cdot 10^{19}$ & 0.009 \\
& & & & 0.41 & $1.1 \cdot 10^{20}$ & 0.016 \\
\hline 5 & 15 & 480 & 200 & 0.60 & $2.3 \cdot 10^{18}$ & 0.019 \\
& & & 0.52 & $3.6 \cdot 10^{18}$ & 0.020 \\
& & & & 0.46 & $8.6 \cdot 10^{18}$ & 0.020 \\
& & & & 0.41 & $1.4 \cdot 10^{19}$ & 0.015 \\
\hline \hline
\end{tabular}

disordered transitional layers of the films, formed in a process of their condensation near the substrate. Besides, in the case of CdTe films, the concentration of the residual admixtures in the material can make $N_{t}=10^{20}$ $10^{21} \mathrm{~m}^{-3}$, which exceeds the sensitivity of the IS method. These admixtures can cause a number of complex admixture-self-defects, that can result in deep levels in BG of the semiconductor [17].

The traps concentration and energy-dependant disorder parameter have been found as dependent on the substrate temperature and the layer thickness. It was also stated that $N_{t}$ and $\sigma$ depend on the physical conditions and the technology of the films production. If the conditions are close to the stable thermodynamic, $N_{t}$ and $\sigma$ parameters decrease. The depth of the traps localization in the studied range $T_{S}$ did not depend on the condensation modes, and their energy spectrum was determined by the admixture defective structure of the material. The calculated data for $N_{t}$ and $E_{t}$ co-relate well with the data obtained from the low-temperature IS method [9] as well as with the data from the literature about the films with similar structural characteristics but surveyed by other methods [17]. Because the films were not alloyed for the purpose, obviously, these LS resulted from the innate defects and the 'innate defect-admixtures' complexes.

Conformity of the traps distribution shape reconstruction in CdTe films and their parameters were checked through the additional direct method. Figs 2 a \& b illustrate the results of the both methods: experimental (dotted curve) and theoretical calculations (solid curve) for the SCLC CVC. As pictured in Figs 2 a, b, these curves almost coincide. The errors for the theoretical CVC curves don't exceed $5 \%$. It proves the viability of the results gained from the research.

The results of the computer modeling and experimental tests prove high informativeness and preciseness of the IS high temperature method. This method can be successfully used to study DT in mono-, polycrystalline and amorphous semiconducting films and massive crystals in both traditional and new (for example, diamond films) semiconducting materials. In fact, the availability of this method for the LS study is limited only by the necessity to produce an injecting contact with a semi-conductor or a dielectric.

\section{References}

1. M. Lampert, P. Mark, Injection currents in solids, "Mir"", Moscow, 1973 (in Russian).

2. K.Cao, V. Huang. Transport of electrons in solids: (in two volumes). V.1, "Mir", Moscow, 1984 (in Russian).

3. D.A. Aronov, Kh.J. Isaev, V.M. Rubinov, M. Tuchiyev. Physical phenomena and their regularities in thin amorphous films of gallium posphide excited by light or fast electron beams // Fizika i tekhnika poluprovodnikov 28(2), pp. 177-197 (1944), in Russian.

4. A.S. Opanasyuk, A.P. Chekalov. Injection spectroscopy of deep trap centers in semiconductor thin films // Visnyk Sums'kogo derzhavnogo universitetu, 1, pp. 29-34 (1994), in Ukranian.

5. J.C. Pfister, Note of interpretation of space-charge-limited currents with traps // Phys. Stat. Sol. 24(1), pp.K15-K17 (1974).

6. S. Nespurek, J.Sworakowski, Use of space-charge-limited current measurement to determine of properties of energetic distributions of bulk traps // J. Appl. Phys. 51(4), pp.2098$2102(1980)$. 


\section{A.S. Opanasyuk et al.: High-temperature injection spectroscopy of deep traps ...}

7. F. Shauer, Zmeskal O., Nespurek S. A new approach to the analysis of steady-state space change limited currents using their activation energies an application to $a-\mathrm{As}_{2} \mathrm{Sl}_{3} / /$ Phys. Stat. Sol. (a). 75(2), pp.531-534 (1983).

8. S.Nespurek, J.Sworakowski, Spectroscopy of traps for current carriers in molecular materials// J. Mol. Electr. 5, pp.7177 (1989).

9. V.O. Lyubchak, A.S. Opanasyuk, N.V. Tirkusova, V.I. Kharchenko, Method of injection spectroscopy for sdudying deep centers in cadmium telluride films // Ukrainski fizicheskii zhurnal 44(6), pp. 741-747 (1999), in Ukrainian.

10. D.Yu. Korol', A.S. Opanasyuk, N.V. Tirkusova, V.I. Kharchenko, Differential method to calculate parameters of deep traps in semi-insulating materials // Matematichne modelyuvannya, 3, pp. 33-37 (1998), in Ukrainian.

11. A.S. Opanasyuk, N.V. Tirkusova, V.I. Kharchenko, A.N. Bobkov. Selfconsistent differential method of injection spectroscopy of deep traps in semi-insulating materials // Visnyk Sums'kogo derzhavnogo universitetu, 1(7), pp. 131138 (1997), in Ukranian.

12. A.S. Opanasyuk, N.V. Tirkusova, High-temperature method of deep trap injection spectroscopy // Visnyk Sums'kogo derzhavnogo universitetu. Seriya; Fizika, matematika, mekhanika, 17, pp. 28-37 (2000), in Ukranian.
13. A.S. Opanasyuk, I.Yu. Protsenko, N.V. Tirkusova, Some features of distribution reconstruction in studying deep states by the method of injection spectroscopy // Zhurnal fizichnykh doslidzhen', 4(2), pp. 208-215 (2000), in Ukrainian.

14. A.I. Zyuganov, S.V. Svechnikov, Injection-contact phenomena in semiconductors, "Naukova dumka", Kiev. 1981 (in Russian).

15. T.Ya. Gorbach, S.V. Svechnikov, P.S. Smertenko ef all. Evolution of current-voltage characteristics inherent to porous silicon after chemical etching // Fizika i tekhnika poluprovodnikov, 31(12), pp. 1414-1418 (1999), in Russian.

16. A.F. Verlan', V.S. Sizikov, Integral equations: method, algorithms, programs. "Naukova dumka", Kiev, 1986 (in Russian).

17. D.V. Korbutyak, S.V. Mel'nichuk, E.V. Korbut, M.M. Borisyuk, Cadmium telluride: impurity-defect states and detector properties, "Ivan Fyodorov", Kyiv (2000), in Ukrainian.

18. Yu. A. Gorokhovatskii, G.A. Bordovskii., Temperature current spectroscopy of higt resistive semiconductors and dielectrics, "Nauka", Moscow (1991), in Russian. 